\title{
ON NONLINEAR FREDHOLM OPERATOR EQUATIONS
}

\author{
BY M. S. BERGER ${ }^{1}$ AND E. PODOLAK
}

Communicated by Hans Weinberger, January 25, 1974

Let $L$ denote a bounded linear Fredholm operator of index $p \geqq 0$ mapping a Hilbert space $H$ into itself with $\operatorname{dim} \operatorname{Ker} L=d$ and $\operatorname{dim}$ coKer $L=d_{*}$. In this note, we consider the solvability of the operator equation

$$
L u+N u=f, \quad f \in H,
$$

where $N$ is a $C^{\mathbf{1}}$ uniformly bounded (nonlinear) mapping of $H$ into itself. If $p=0$ and $N$ is compact, the solvability of (1) is generally studied by means of the Leray-Schauder degree [1]. However for $p>0$, more general methods of study are required, since A. Svarc [2] showed the homotopy classes of (singularity-free) mappings $L+N$ restricted to the unit sphere in $H$ are in (1-1) correspondence with the stable homotopy group $\pi_{n+p}\left(S^{n}\right)(n>p+1)$. More recently, L. Nirenberg [3] studied criteria for solvability of coercive boundary value problems for elliptic systems (defined on bounded domains or compact manifolds) of the form (1) (with $N(u)$ satisfying additional compactness and asymptotic properties). He showed that the criteria could be expressed in terms of the nontriviality of the stable homotopy class of a certain continuous mapping $\mu$ of the $(d-1)$-sphere $S^{d-1} \subset \operatorname{Ker} L$ into the $\left(d_{*}-1\right)$-sphere $S^{d_{*-1}} \subset \operatorname{coKer} L$. Nirenberg remarks that this criterion is difficult to apply since, in general, it is not known how to compute the stable homotopy class of $\mu$.

Here we take up this solvability problem from a simple Hilbert space point of view and sharpen the results just mentioned in several respects. First, we remove the compactness requirement for the operator $N$, thus allowing the applicability of our results to elliptic systems (that can be put in the form (1)) defined over unbounded domains and noncompact manifolds. More importantly, we derive a criterion for solvability of (1) based on the nontriviality of the homotopy class [ $\eta]$ of a mapping $\eta$ of $S^{d-1} \rightarrow S^{d_{*-1}}$ (analogous to the mapping $\mu$ of [3]), provided $L$ "dominates" $N$ in the sense described below. More generally, if $L$ "dominates" $N$ apart from a finite-dimensional subspace of dimension $m$ (as always occurs

AMS (MOS) subject classifications (1970). Primary 47H15, 35J60.

${ }^{1}$ Research partially supported by an AFOSR grant 73-2437 and an NSF grant. 
in the cases described in [3]), our criterion is based on the nontriviality of the homotopy class of the $m$ th iteration of the (Freudenthal) suspension homomorphism $E^{m}[\mu]$. In applications to elliptic systems, the integer $m$ is found by relating the spectrum of $L$ to the "size" of $N$. In case $L$ is selfadjoint, we find a necessary and sufficient condition for the solvability of (1) that implies the openness of the range of $L+N$. Full details of the proofs will be given elsewhere.

1. The solvability result. In accord with [3], we make the following assumptions on the operator $N$, and its Fréchet derivative $N^{\prime}(u)$ :

(I) $\left\|N^{\prime}(u)\right\| \leqq c$, where $c$ is some constant independent of $u$;

(II) $\eta(a)=\lim _{r \rightarrow \infty} P^{*}\{N(r a+x)-f\}$ exists uniformly for $\|x\|$ uniformly bounded and $a \in \partial \Sigma=\operatorname{Ker} L \cap\{w \mid\|w\|=1\}$ where $\eta(a) \neq 0$. Here $P^{*}$ is the canonical projection of $H$ onto coKer $L$.

Under these assumptions we prove

THEOREM 1. Suppose that apart from a finite-dimensional subspace $W=\operatorname{Ker} L \oplus V$ of $H, L$ dominates $N$ in the sense that

$$
\|L w\| \geqq(c+\varepsilon)\|w\| \quad \text { for some fixed } \varepsilon>0 .
$$

Then, if $\operatorname{dim} V=m$, equation (1) is solvable provided the mth iterate $E^{m}[\tilde{\eta}]$ of the suspension homomorphism $E$ of the homotopy class [ $\tilde{\eta}]$ of $\tilde{\eta}=\eta|| \eta \mid$ is a nontrivial element of $\pi_{m+d-1}\left(S^{m+d_{*}-1}\right)$. In particular, (1) is solvable provided $[\tilde{\eta}]$ is nontrivial if either $m=0, p=0$, or more generally $E^{m}$ is an isomorphism of $\pi_{d-1}\left(S^{d_{*}-1}\right)$ into $\pi_{m+d-1}\left(S^{m+d_{*}-1}\right)$.

SKETCH OF PROOF. We begin by replacing equation (1) by the system

$$
\begin{aligned}
L^{*} L u+L^{*} N u-L^{*} f & =0, \\
P^{*}(N u-f) & =0 .
\end{aligned}
$$

This pair implies that $A u=L u+N u-f \in \operatorname{Ker} L^{*} \cap\left[\operatorname{Ker} L^{*}\right]^{\perp}$, so that (1) is satisfied if (3) holds for some $u \in H$. The converse is immediate.

To study the solvability of (3a), we utilize hypothesis (I) to reduce (3a) to a finite-dimensional system. To this end, let $H=\operatorname{Ker} L \oplus V \oplus H_{1}$ where $H_{1}=W^{\perp}$ and denote by $P_{1}$ the canonical projection of $H$ onto $H_{1}$. Then, for $w \in H_{1}$ and arbitrary $x \in H$, (2) implies $\left(L^{*} A^{\prime}(x) w, w\right) \geqq \varepsilon\|w\|^{2}$, where $A^{\prime}(x)$ denotes the Fréchet derivative of $A(x)$. Consequently by Hadamard's theorem (see [4, pp. 16-18]), $P_{1} L^{*} A(x+w)$ is a global homeomorphism of $H_{1}$ onto itself. Now (3a) is automatically satisfied on Ker $L$. Hence if a tentative solution of the system (3) is written $u=z+y+w$ with $z \in \operatorname{Ker} L$, $y \in V$, and $w \in H_{1}$, it suffices to solve the finite-dimensional system

$$
L^{*} L y+P_{v}\left(L^{*} N(u)-f\right)=0, \quad P_{v} \equiv \operatorname{proj} H \rightarrow V .
$$


Now we consider the finite-dimensional system (3b) and (4), and note that on a sufficiently large sphere in $W$, the mapping so defined is homotopic to the mapping

$$
\Phi(y, z)=\left(y, P^{*}(N(z)-f)\right) .
$$

This fact follows since the uniform boundedness of $N(u)$ over $H$ yields uniform a priori bounds for $\|w\|$ and $\|y\|$ satisfying (3). Now, let $\eta(a)$ be the mapping of $S^{d-1} \rightarrow \operatorname{coKer} L$ defined in (II), and $\tilde{\eta}$ be the associated normalized mapping. Then we observe that the homotopy class $[\tilde{\Phi}]$ of the normalized mapping $\tilde{\Phi}=\Phi /|\Phi|$ regarded as a mapping from $S^{m+d-1} \rightarrow S^{m+d_{*}-1}$ satisfies $[\tilde{\Phi}]=E^{m}[\tilde{\eta}]$, where $E^{m}$ is the $m$ th iterate of the Freudenthal suspension homomorphism. Thus the theorem follows.

2. Necessary and sufficient conditions for solvability. As an application of Theorem 1 (in case $L$ is selfadjoint), we prove

TheOREM 2. Suppose $L$ is selfadjoint, $N$ satisfies the hypotheses of Theorem 1 , and in addition, for all positive numbers $r$

$$
(N(u), a)<(\phi(a), a)
$$

with

$$
\phi(a)=\lim _{r \rightarrow \infty} P^{*} N(r a), \quad a \in \partial \Sigma \quad \text { and } \quad x \perp \text { Ker } L .
$$

Then, (a) a necessary and sufficient condition for the solvability of (1) is

$$
(f, a)<(\phi(a), a) \text { for all } a \in \partial \Sigma ;
$$

and (b) the mapping $L+N$ has open range.

Proof of (a). The necessity of (7) follows immediately from (6) and the selfadjointness of $L$. The sufficiency of (7) follows from Theorem 1 and hypothesis (II), since in this case index $L=0$ and for $r$ sufficiently large the Brouwer degree

$$
\operatorname{deg}(P N(z), P f,|z|<r)=\operatorname{deg}(\phi, f,|z|<r)=1,
$$

where $P$ denotes the canonical projection of $H$ onto $\operatorname{Ker} L$.

Proof of (b). Let $f_{0} \in \operatorname{Range}(L+N)$. Then, to prove $L+N$ has open range, it suffices to show that $S_{\varepsilon}=\left\{f \mid\left\|f-f_{0}\right\|_{H}<\varepsilon\right\}$ (for some $\varepsilon>0$ ) also lies in $\operatorname{Range}(L+N)$. This last fact follows immediately from (7) for $f \in[\operatorname{Ker} L]^{\perp} \cap S_{\varepsilon}$ for any $\varepsilon$ and for $\left\|P\left\{f-f_{0}\right\}\right\|$ sufficiently small, by virtue of the strict inequality in (7) and the finiteness of $\operatorname{dim} \operatorname{Ker} L$.

REMARK. Theorems 1 and 2 are readily applicable to the study of boundary value problems of semilinear elliptic systems defined on manifolds or domains in Euclidean space $\boldsymbol{R}^{N}$. In such cases (2) of Theorem 1 will always be satisfied for some finite-dimensional subspace $W$ provided the spectrum of the associated linear differential operator $L$ is discrete.

To find relations between the solutions of (1), we prove 
THEOREM 3. Suppose that in addition to the hypotheses of Theorem 2, $N$ is a completely continuous operator. Then, if $f \in \operatorname{Range}(L+N)$ (except for a possible set of the first Baire category) (i) each solution $x$ of (1) is nondegenerate (i.e. $L+N^{\prime}(x)$ is an invertible linear operator), (ii) the solutions of (1) are finite in number, and (iii) if the Fréchet derivative of $N$ is selfadjoint, and off $W, L+N^{\prime}>0$, while $N^{\prime}<0$ on $H$, the following Morse inequalities hold, where the $M_{i}$ denote the number of solutions of (1) of Morse index $i$ and $n=\operatorname{dim}[W r\{L \leqq 0\}]$ :

$$
\begin{gathered}
M_{n} \geqq 1, \quad M_{n+1}-M_{n} \geqq-1, \\
M_{n+2}-M_{n+1}+M_{n} \geqq 1, \cdots, \quad \sum_{i=n}^{\operatorname{dim} W}(-1)^{i} M_{i}= \pm 1 .
\end{gathered}
$$

SKETCH OF PROOF. We first note that the solvability of (1) implies (by virtue of Theorem 2) (*) the uniform boundedness of any sequence $\left\{u_{n}\right\}$ for which $\left\|L u_{n}+N u_{n}-f\right\| \rightarrow 0$. This implies (by the inverse function theorem and Smale's version of Sard's theorem) that, apart from a possible exceptional set of first Baire category, the solutions of (1) are nondegenerate and isolated. The complete continuity of $N$ and the Fredholm property of $L$ yield the finiteness of these solutions. Moreover, by virtue of $(*)$, the functional $I(u)$ defined by the relation $I^{\prime}(u)=L u+N u-f$ satisfies the Palais-Smale condition (C) on $H$. Consequently, a modification of Smale's version of Morse theory on Hilbert space establishes the relations (8).

AdDED IN PROOF. Theorem 1 is true for mappings $L+N$ of a Banach space $X$ into a Banach space $Y$ provided hypothesis $(I)$ is altered to read $\left\|P_{1} N^{\prime}(u)\right\| \leqq c$ where $P_{1}$ is a projection of $Y$ onto $L(X / W)$. Moreover, examples show that Theorem 1 is sharp in the senses that (i) the homotopy class $[\tilde{\eta}]$ may be nontrivial and equation not solvable, (ii) the stable homotopy class of $\tilde{\eta}$ may be trivial, yet equation (1) is solvable by the criterion given in Theorem 1 with $m \neq 0$.

\section{BIBLIOGRAPHY}

1. J. Leray and J. Schauder, Topologie et équations fonctionelles, Ann. Sci. École Norm. Sup. 51 (1934), 45-78.

2. A. S. Švarc, The homotopic topology of Banach spaces, Dokl. Akad. Nauk SSSR 154 (1964), 61-63 =Soviet Math. Dokl. 5 (1964), 57-59. MR 28 \#3309.

3. L. Nirenberg, An application of generalized degree to a class of nonlinear problems, Colloq. Analyse Fonctionelle, Liège, 1972, pp. 57-74.

4. J. Schwartz, Nonlinear functional analysis, Gordon and Breach, New York, 1969.

Belfer Graduate School, Yeshiva University, New York, New York 10033

Current address (E. Podolak): Department of Mathematics, Princeton University, Princeton, New Jersey 08540 